\title{
Choose Topic Research Resources Effectively in the Network Training
}

\author{
Lujun $\mathrm{Fu}^{1, \mathrm{a}}$, Lan Dou ${ }^{2, \mathrm{~b}}$ \\ ${ }^{1}$ School of Harbin Normal University, Harbin 150025, China \\ ${ }^{2}$ School of Harbin Normal University, Harbin 150025, China \\ afulujun0512@163.com, ${ }^{b} 1206556030 @ q q . c o m$
}

\begin{abstract}
Keywords: Kindergarten teacher workshop; Localizing research; Research resources; Topic
\end{abstract} research; Research way.

\begin{abstract}
In the national training project (2015) of kindergarten teacher workshop seeded teacher training, localizing research resources are based on a principle, which includes adaptability, integration, practice and generativity. Combining social needs with regional education condition, an exploratory and pre-applied plan of topic research were raised up after dealing with the difficulty of localizing research resources selection. Through the empirical study, it can finally come to the conclusion. The plan was to occupy the resources for establishing a topic, screen the resources, to run through the topic, and compact the resources to sublimate the level of the topic. Throughout the transformation of basic resources, procedural resources, and conclusive resources, students can solve practical problems and improve specialty literacy by training on the research way of independent, groups or teams.
\end{abstract}

\section{Introduction}

In the 1960s, "workshop" was applied to urban projects by Lawrence Halprin in United States. That made it become a way of communicating, discussing, participating, innovating, and finding methods on problems [1]. "Kindergarten teacher workshop "made the idea of "workshop" into teacher training, which changed the traditional training approach. That was based on expert lectures as the main components, centered on subject contents, carried by the short term training and driven by training task. By " Kindergarten teacher workshop ", a new mode was forming up, which was based on self-training as the main components, centered on collective training, carried by online binding downline, driven by teacher professional demand, and combined with practical factors to being new information network training platform, such as self-doing and mutual cooperation.

\section{The basic principle of effectively selecting for training resources}

\subsection{Adaptability Principle}

As a starting point to demand the trainees' real needs, the traditional idea that was 'offer what I have 'was changed to 'offer what teacher needs and what teacher lacks of' [2]. On the vertical angle, from the overall goal to the layer goal, the resources should be submitted to the research topics, and met the training objectives, and be beneficial to training evaluation. On the horizontal angle, resources selection should be fundamental on individual development of trainees and meet the needs of students in the topic training.

\subsection{Integration Principle}

Teacher knowledge is an inextricably linked system. All kinds of knowledge interact together and integrate each other. It promotes the accumulation and construction of theoretical knowledge and practical knowledge of teachers [3]. From the macro point of view, the resources selection has an overall effect that it will lead the whole body. The degree that the resources, the target and the students' needs are matched directly influences on the overall effect of the research. From the microscopic point of view, integration of resources elements is the only way to achieve the overall effect of resources than sum of that. It also helps students establish a global awareness and carry out systematic and in-depth training. 


\subsection{Practical Principle}

The practical principle is based on the action. That means learning in action, training in action, and improving in action. From the perspective of epistemology, resources training come from the teaching practice. It can help trainees understand practical resources further by screening, sorting, summarizing and applying on. Meanwhile, it guides on practice as the resources are generated continuously. The resources generated on the progress of the students "in the field" interaction are evolved to be a bright spot in the training, which overcomes problems that only media content presentation is paid attention to in the progress of training and shortcoming of communication was ignored in the dynamic progress.

\subsection{Generativity Principle}

Bloom proposed: "the reason why the teaching class becomes the teaching art was the unpredictable nature of the classroom implementation." That was the generativity principle. Contingency and differences was the main feature of generative teaching [4]. Sustainable development of the resources is the basis and premise of procedural resources and conclusive resources. At the same time, inspiration of resource leads the students to interact with environment and others. Finally, to resources, creative choice and creative use and creative generation all results from the multivariate integration mechanism of localizing research resources, which are also the most valuable resources of that.

\section{The dimension of effectively selecting the training resources}

\subsection{Social needs determine the target orientation of research resources}

Taylor put forward the target mode of curriculum development, namely "to determine the target of education", "choice of educational experience", "the organization of the educational experience", "education evaluation plan" [5]. Training objectives are to determine the premise of resource selection. In order to make target localized, on the basis of social needs, clear the initial period of the training. Then, pay attention to the training of practice choices, purposeful, plan selection and organization resources, follow the teachers' professional growth rule, realize the resource and member of two-way role and help the students to realize the professional development.

\subsection{The content of the educational situation in the region determines the content of the training resources}

Canadian scholar Fullan has proposed the implementation of the changes "25\%:75\% rule", it is that $25 \%$ of the scheme and $75 \%$ condition of effective implementation scheme[6]. The regional education is the core of the localization resources selection, mainly including the status of the regional education and the status of the personal education, the investment funds of education, the construction of the team, the administrative management, etc., The choice of resources presents macro radiation diffusion, the width of the choice of resources, the individual professional ability and the influence resources choice depth and the thickness.

\subsection{The research teams determine the process of the training resources}

The research teams preinstall to study what and how to study, according to the "understanding" for the students. The choice of resources relies on understanding. And the different cognitive results determine the choice of resources, such as the perspective of the choice of process oriented differences. National training trial standards emphasize the resources of the local conditions, education and suitability, training team from the training plan establishment, the theme of supposition, program development, theme training and evaluation.

\section{Network platform topic of pre-training plan for the default}

\subsection{Occupy resources and locate the topic}

The topic of seminary is origin from practical resources. Teachers can make demands centralized rapidly by teaching, and determine the topics of the seminars. The practice resources, from bottom to up, had pertinence and applicability, which was conducive to the mobilization and the enthusiasm of 
trainees, and achieved multiple understanding. Besides, research topics should be based on system analysis of resource, the design, from top to down, made the topics own theoretical foundation and operability. In general, the practical resources, from bottom to up, were the foundation of resources choice, while that, from top to down, was the mainstream of resources choice.

\subsection{Screen resources and run through the topic}

4.2.1 From the macro perspective to research the resource connection topic

Macro selection of resources refers to the overall process of resource based on training. Research resources are divided into the basic resources, the procedural resources and conclusive resources. They are always throughout the independent research period, group training period and collective research period.

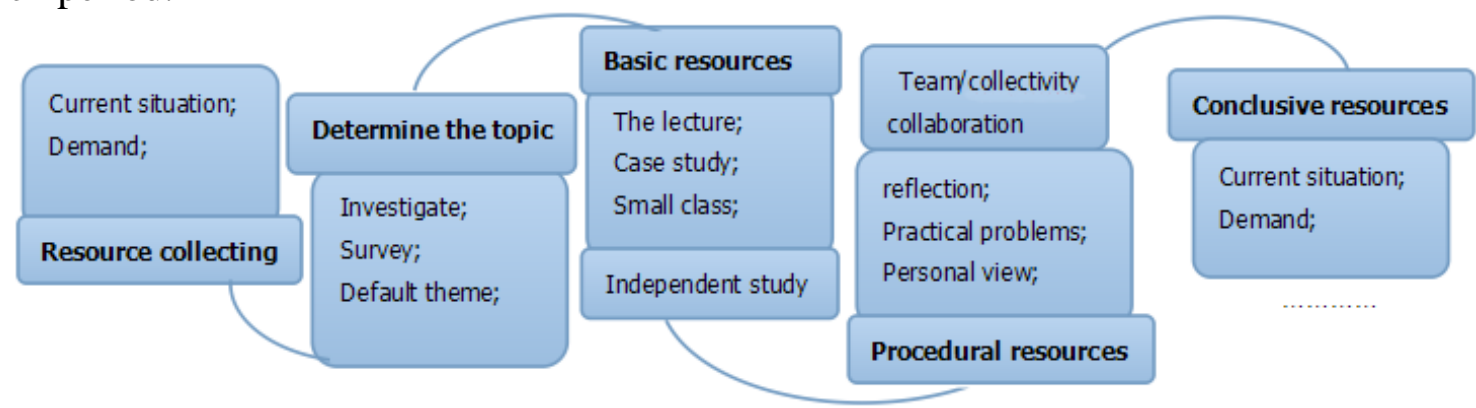

Fig. 1 Resource connection topic Icon

\subsubsection{From the medium perspective to research the resources generation}

The medium selection of resources means that the choice of resources must be based on the transitivity between research resources and resources. The topic research is divided into the independent research period, the group cooperation research period and the collective research period. The degree of periodical resources change and the degree of research is positively related, as shown in Figure 2.

Independent phase $\quad$ Group phase
Basic resources
The lower level of independent study resources Group, the lower level of collective study

Fig. 2 Resource transformation diagram

First of all, independent research is the primary period of a topic research. Students study fundamental resources. The deeper the independent research studied, the higher the extent of the basic resources transformed into procedural resources is. But, the lower level of training, the higher the initialized degree of resource is. Secondly, group research is the middle period in the topic research. Students achieve methods to solve problems and rich perspective through dialogue, exchanges and sharing in the group. The deeper the group research is proceeded to, the higher the degree of rational knowledge of basic resources transformed into procedural resources is. On the contrary, the lower the perceptual knowledge of one's resources is. Finally, collective research is the late period in the topic research. All students can view a spokesman for the discussion of the whole process. Through collective thinking collision, they can understand special subject deeply. And solutions may be got. In this period, the more intense the collective study is, the higher the value of procedural resources transformed into conclusive resources.

4.2.3 From the micro perspective to research resource dynamic development

The micro selection of resources refers to that the resources choice must be based on research change in the period. Dynamic process of continuous transformation of basic resources, procedural resources and conclusive resources in this period is analyzed in this paper. Firstly, the basic resource is the "root" of the topic research. It refers to obbligato resources, such as a line of teacher's classroom, 
classic case, the seminar, interpretation, etc. Secondly, the procedural resource is the "stem" of the topic research. In any period, any research forms all can induce the formation of the procedural resources. Finally, conclusive resource is the "fruit" of the topic research. It is an intelligence gathering. And it reflects the problems that students encounter in the practice, which provides the basis for late professional diagnosis.

\subsection{Concise resources and sublimate the topic}

Conclusive resource is the key to the sublimation of topic. There are two research topics after sublimation. One is a lot of summary of the practical experience; the other is the new formed research topics. From possessing resources and analyzing topic to providing resources in the different periods, it runs through the topic. In the collective research, conclusive resources are got, which provide material for selection of next topic research. Spiral resource generation mode makes students use the research platform of resources. It promotes the research to go.

\section{Conclusions}

Kindergarten teacher workshop is a new teacher training mode. The topic of network platform is in the testing period. There are still many problems in this research topic. For example, how to realize the localization of the training resources, and how to realize the resources to be maximum used. These problems depend on subsequent practice and reflection. We believe that workshop training mode will earn more return with minimum expenditure in the shortest time in the future, and meet the teacher professional development needs and specialized requirements.

\section{References}

[1]. Ge Hua, The design and application of "Practice teaching workshop", Education Theory and Practice, vol.31, pp.45-47, June 2011 (In Chinese).

[2]. Xu Weiliang, "National training plan" effective curriculum development five step in Hunan Province replacement full-time training project curriculum setting for patients, Chifeng University Journal (NATURAL SCIENCE EDITION), vol.28, pp. 171-174, May 2012 (In Chinese).

[3]. Song Jun, Dalian Ganjingzi District of teacher training curriculum development and implementation, Liaoning: Liaoning Normal University, 2013, pp. 1-56 (In Chinese).

[4]. Zhang Jianlin, The generative principle of effective teaching in middle school geography classroom, Course Education Research, pp.170-171, May 2014 (In Chinese).

[5]. Chen Min, etc, Enlightenment of the post-modernist Curriculum on the reform of biology teaching in Senior Middle School (middle school), pp.102-103, 2010 (62) (In Chinese).

[6]. Darin Bohr, Education reform limits, translated by Liu Chenghui. Chongqing: Chongqing University Press, 1991. 